\title{
Wide-Ranging Effects on the Brain Proteome in a Transgenic Mouse Model of Alzheimer's Disease Following Treatment with a Brain- Targeting Somatostatin Peptide
}

Fadi Rofo, Friederike A. Sandbaumhüter, Aikaterini Chourlia, Nicole G. Metzendorf, Jamie I. Morrison, Stina Syvänen, Per E. Andrén, Erik T. Jansson, and Greta Hultqvist*

Cite This: ACS Chem. Neurosci. 2021, 12, 2529-2541

Read Online

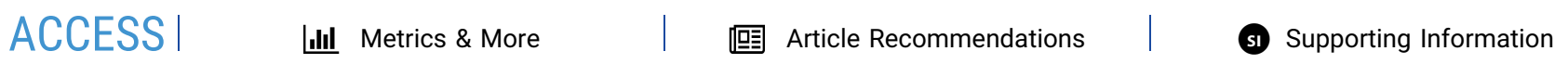

ABSTRACT: Alzheimer's disease is the most common neurodegenerative disorder characterized by the pathological aggregation of amyloid- $\beta(\mathrm{A} \beta)$ peptide. A potential therapeutic intervention in Alzheimer's disease is to enhance $\mathrm{A} \beta$ degradation by increasing the activity of $A \beta$-degrading enzymes, including neprilysin. The somatostatin (SST) peptide has been identified as an activator of neprilysin. Recently, we demonstrated the ability of a brain-penetrating SST peptide (SST-scFv8D3) to increase neprilysin activity and membrane-bound $\mathrm{A} / 42$ degradation in the hippocampus of mice overexpressing the $\mathrm{A} \beta$-precursor protein with the Swedish mutation (APPswe). Using LC-MS, we further evaluated the anti-Alzheimer's disease effects of SST-scFv8D3. Following a triple intravenous injection of SST-scFv8D3, the LC-MS analysis of the brain proteome revealed that the majority of downregulated proteins consisted of mitochondrial proteins regulating fatty acid oxidation, which are otherwise upregulated in APPswe mice compared to wild-type mice. Moreover, treatment with SST-scFv8D3 significantly increased hippocampal levels of synaptic proteins regulating cell membrane trafficking and neuronal development. Finally, hippocampal concentrations of growth-regulated $\alpha$ (KC/GRO) chemokine and degradation of neuropeptide-Y were elevated after SST-scFv8D3 treatment. In summary, our results demonstrate a multifaceted effect profile in regulating mitochondrial function and neurogenesis following treatment with SST-scFv8D3, further suggesting the development of Alzheimer's disease therapies based on SST peptides.

KEYWORDS: Alzheimer's disease, amyloid- $\beta$, proteomics, somatostatin, SST-scFv8D3, LC-MS

\section{INTRODUCTION}

Alzheimer's disease is a progressive and multifactorial neurodegenerative disorder. It is also the most common cause of dementia. In the brain of Alzheimer's patients, extracellular amyloid aggregates and intracellular neurofibrillary tangles are formed, leading to synaptic toxicity and neuronal death. ${ }^{1}$ The pathological amyloid aggregation is formed by a 36-43 amino acid long peptide called amyloid- $\beta(\mathrm{A} \beta)$. The latter is generated by enzymatic digestion of the transmembrane protein, amyloid precursor protein (APP). Familial mutations in APP, such as the Swedish mutation (APPswe) (KM670/ $671 \mathrm{NL}$ ), increase $A \beta$ production and lead to early onset of Alzheimer's disease. ${ }^{2}$ Accumulation of $\mathrm{A} \beta$ can also be attributed to the decline in the activity and/or levels of the $\mathrm{A} \beta$-degrading enzymes, including neprilysin. ${ }^{3}$

Neprilysin is a membrane-bound zinc-metallopeptidase with a large extracellular domain containing the catalytic site. The enzyme has displayed the ability to degrade $\mathrm{A} \beta$ but not its precursor APP. ${ }^{4}$ The protein levels of neprilysin are downregulated during aging and Alzheimer's disease, both in transgenic mouse models of Alzheimer's disease and in post- mortem human brains. ${ }^{5,6}$ Accordingly, molecules that enhance the activity or levels of neprilysin are considered as potential therapeutic options for the treatment of Alzheimer's disease.

A major activator of neprilysin is somatostatin (SST), a cyclic neuropeptide involved in processes of neuromodulation and cell proliferation through its interaction with SST receptors (SSTRs). ${ }^{7}$ Importantly, SST has been identified as a major activator of neprilysin. Treatment of primary neuronal cultures with this peptide has been associated with enhanced neprilysin activity and decreased $\mathrm{A} \beta 42$ concentration. ${ }^{8}$ In addition to its role in the proteolytic degradation of $\mathrm{A} \beta$, the levels of SST peptide are downregulated in the early stages of Alzheimer's disease, especially in the hippocampus and neocortex, ${ }^{9,10}$ areas within the brain from where $\mathrm{A} \beta$ pathology

Received: May 11, 2021

Accepted: June 15, 2021

Published: June 25, 2021 


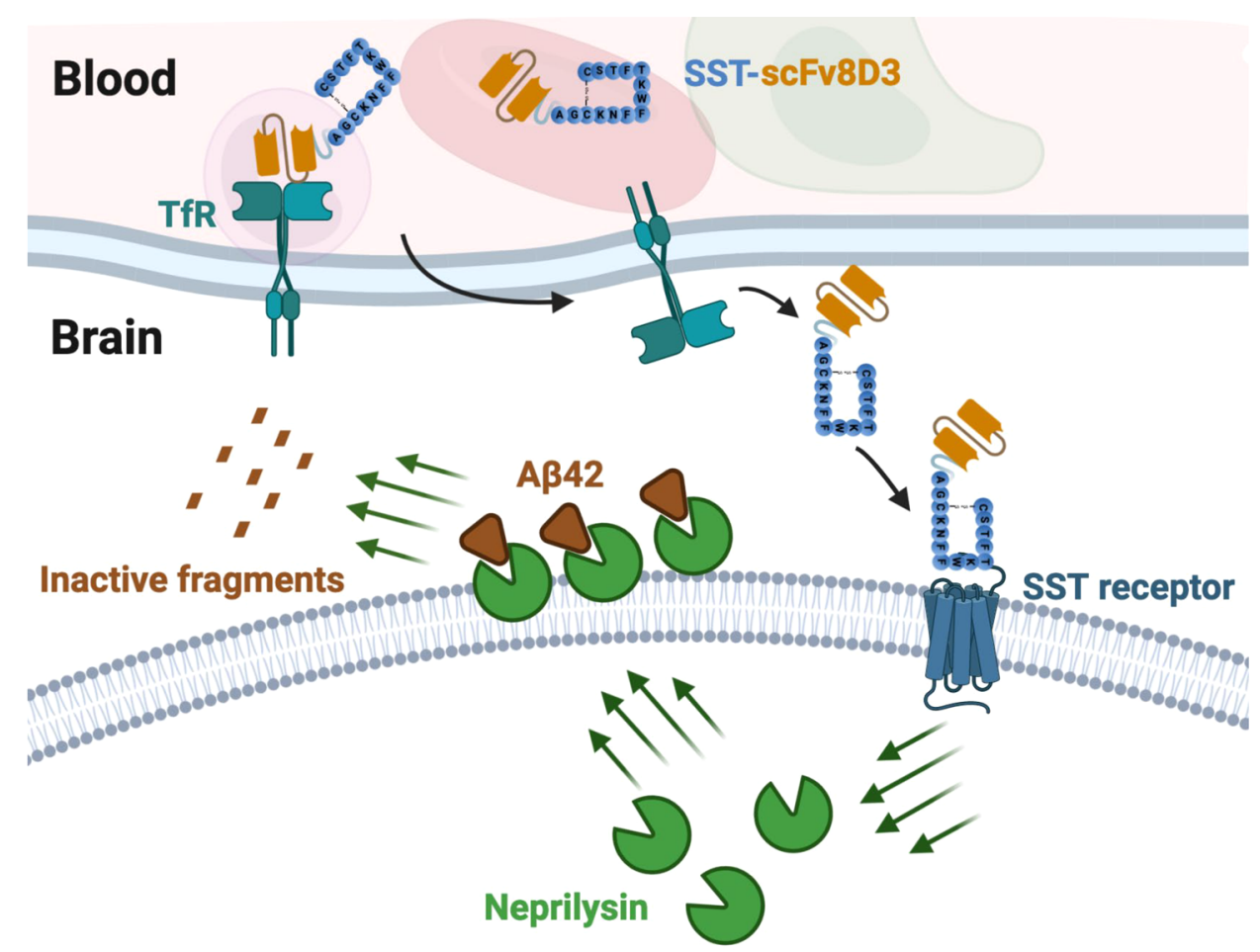

Figure 1. Intravenously injected SST-scFv8D3 passes from blood to brain by binding to the transferrin receptor (TfR). In the brain, SST-scFv8D3 interacts with SST-receptors. This interaction causes a series of downstream events, pushing neprilysin toward the membrane of the neurons, where it can degrade membrane-bound $\mathrm{A} \beta 42$ into inactive fragments.

initially progresses. ${ }^{11}$ Colocalization of SST and its receptors with $\mathrm{A} \beta$ pathology in the brain and its ability to activate neprilysin suggest the use of the peptide as a promising therapeutic option in Alzheimer's disease.

We have previously shown the high brain uptakes and long plasma half-life of the 14 amino acid-long SST peptide recombinantly expressed with a protein-based blood-brain barrier (BBB) transporter when injected intravenously. ${ }^{6}$ The scFv8D3 transporter of the SST peptide binds to the mouse transferrin receptor (TfR), which has previously been used to increase brain uptake of protein-based drugs used in the diagnosis and treatment of Alzheimer's disease. ${ }^{12-14}$ The recombinantly expressed protein (SST-scFv8D3) increased both the neprilysin concentration and activity within the brain of APPswe mice. In addition, our previous study displayed a significant reduction in the concentration of the membranebound aggregation prone $\mathrm{A} \beta 42$ in the hippocampal area after triple intravenous injections of $1 \mathrm{mg} / \mathrm{kg} \mathrm{SST-scFv8D} 3 .^{6}$ While these effects highlighted SST-scFv8D3 as a potential therapeutic option in the treatment of early Alzheimer's disease, changes in the levels of other proteins in the brain resulting from such treatment need to be addressed. Regarding binding of SST-scFv8D3 to SSTRs, the subsequent enhancement of neprilysin levels and hippocampal $\mathrm{A} \beta 42$ degradation is likely associated with significant changes in the levels of other as of yet unknown proteins involved in Alzheimer's disease pathology (Figure 1).

In this study, we aimed to integrate anti-Alzheimer's-diseaserelated effects from the treatment with SST-scFv8D3 with untargeted analysis of the brain proteome in APPswe mice. In detail, we evaluated protein levels in the hippocampus, the rest of cerebrum, and the cerebellum, following treatment with SST-scFv8D3. Furthermore, a transgenic study of untreated
APPswe and wild-type (WT) mice was conducted to evaluate whether SST-scFv8D3 treatment shifts the altered proteins toward wild-type levels or not. Interesting targets identified by the proteomic analysis with LC-MS were further analyzed by Western blot. In addition to the results generated from LCMS analysis, we aimed to study the effects of such treatment on inflammation, as the latter plays a major role in the pathology of Alzheimer's disease. ${ }^{15}$ In our previous study, no significant differences were detected in the concentration of interleukins after treatment with SST-scFv8D $3 .{ }^{6}$ However, the response of the immune system to such treatment could include processes that leave interleukin levels unaltered. Therefore, a multiplex pro-inflammatory cytokine panel analysis was conducted to quantify the concentration of other inflammatory markers, such as growth-regulated $\alpha(\mathrm{KC} /$ GRO) chemokine, in response to SST-scFv8D3 treatment. Finally, brain concentrations of peptide substrates for neprilysin other than $\mathrm{A} \beta$ were quantified following treatment with SST-scFv8D3, to evaluate the downstream effects of enhanced neprilysin activation.

\section{RESULTS}

To evaluate the effects of SST-scFv8D3 treatment on the brain proteome, two LC-MS studies were conducted. In the first study, we compared the brain proteome of APPswe mice treated with SST-scFv8D3 with that of APPswe mice treated with PBS; hereafter, this is referred to as the treatment study. The second study aimed at comparing the brain proteome of untreated APPswe mice with that of WT controls; hereafter, this is referred to as the transgenic study. A schematic illustration of the workflow can be found in Figure 2 .

LC-MS Analysis of the Hippocampal Area, Rest of Cerebrum, and Cerebellum (Treatment Study). The 
(1) Treatment study

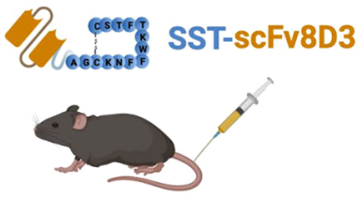

APPswe $(n=7)$
(2) Transgenic study

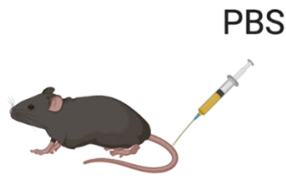

APPswe $(n=7)$

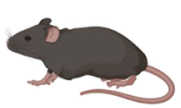

Wild-type $(n=5)$

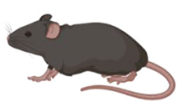

APPswe $(n=4)$

(3) Brain isolation, region dissection and tissue processing

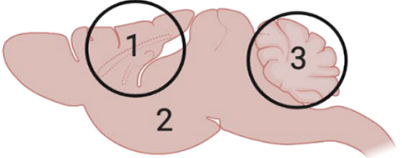

1) Hippocampal area

2) Rest of cerebrum

3) Cerebellum

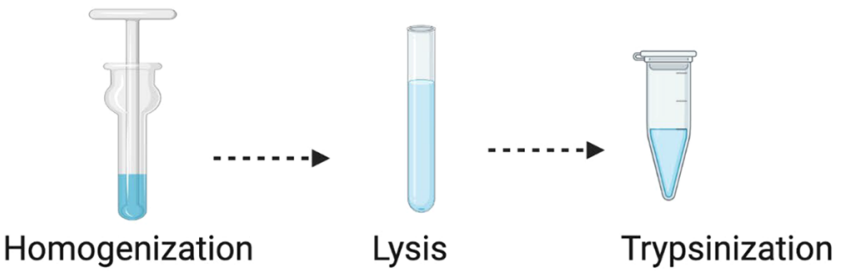

\section{(4) Sample analysis}

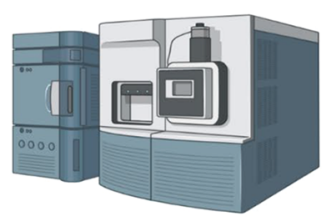

LC-MS

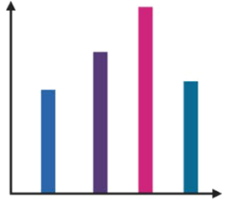

Data processing

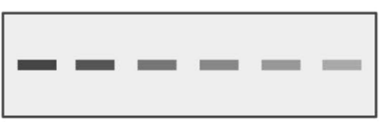

Western blot

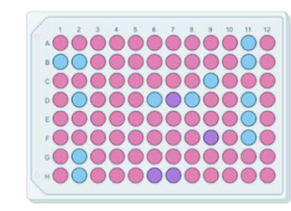

Neuropeptides \& chemokines quantification

Figure 2. Schematic workflow of the study. For the treatment study, eight-month-old transgenic mice with an Alzheimer's-like pathology (APPswe) were divided into two groups. The first group $(n=7)$ was injected intravenously with $1 \mathrm{mg} / \mathrm{kg}$ SST-scFv8D3 every $36 \mathrm{~h}$ (three injections in total). The second group $(n=7)$ was injected intravenously with PBS at the same time points as those of the SST-scFv8D3 group. Mice were euthanized $24 \mathrm{~h}$ after the last injection. For the transgenic study, C57Bl/6 wild-type mice $(n=5)$ and APPswe mice $(n=4)$ at the same age as the mice included in the treatment study were used. Brains were isolated and dissected into three parts: hippocampal area, rest of cerebrum, and cerebellum. This was followed by homogenization with Tris buffer, lysis with HEPES/Urea buffer, and trypsinization, followed by analysis with LC-MS, Western blot, and quantification of neuropeptides and chemokines.

proteome of the hippocampus, rest of the cerebrum, and cerebellum, from seven SST-scFv8D3 treated and seven PBS treated transgenic APPswe mice, were analyzed with LC-MS. In total, 1869 proteins could be quantified across the 42 samples (Table S1). In the hippocampal area, the levels of 55 proteins were significantly increased, and 105 proteins were significantly decreased in the SST-scFv8D3 treated APPswe mice compared to those of the PBS injected group (Figure 3A). No gender-specific differences were detected between SST-scFv8D3 treated and PBS treated APPswe mice.

No significant differences were found in either the cerebellum or in the rest of the cerebrum (Figure 3B,C), which is in line with our previous observations. ${ }^{6}$ Mitochondrial and endoplasmic reticulum resident proteins represented the majority of proteins with significantly lower levels in the hippocampus of SST-scFv8D3 treated APPswe mice (Table S2 and Figure S1). Synaptic proteins involved in regulating trafficking across the cell membrane and neuronal development represented the majority of the proteins with significantly higher levels in the hippocampus of SST-scFv8D3 treated APPswe mice (Table S3 and Figure S1). The top 10 biological processes enriched due to changes in hippocampal protein levels of APPswe mice after treatment with SST-scFv8D3 are illustrated in Table 1. In addition, the altered proteins were mainly associated with mitochondria as displayed in the top 10 cellular components in Table 2.

LC-MS Analysis of the Hippocampal Area and Rest of Cerebrum (Transgenic Study). In order to evaluate whether SST-scFv8D3 treatment shifts the altered proteins toward WT levels, untreated APPswe and WT mice of the same age as the above-mentioned APPswe treated groups were compared in the transgenic study. Hippocampus and rest of cerebrum from four APPswe and five WT mice were analyzed with LC-MS as described above (Figure 2). In total, 2487 proteins could be detected across the 18 samples (Table S4). In the hippocampal area, the levels of 1283 proteins were significantly altered (Figure 3D). In the rest of the cerebrum, the levels of 1379 proteins were significantly altered (Figure $3 \mathrm{E})$. Out of these, 922 proteins were significantly altered in both regions. Amyloid- $\beta$ precursor protein (A4_mouse) was among the proteins with significantly increased levels in APPswe compared to those of WT mice in both regions (Table S4). Other proteins like the synaptic protein syntaxin1A (STX1A_mouse) and the transforming growth factor $\beta-1$ (TGFB1) protein (TGFB1_mouse) represented some of the proteins with the most significant increase in the brains of 


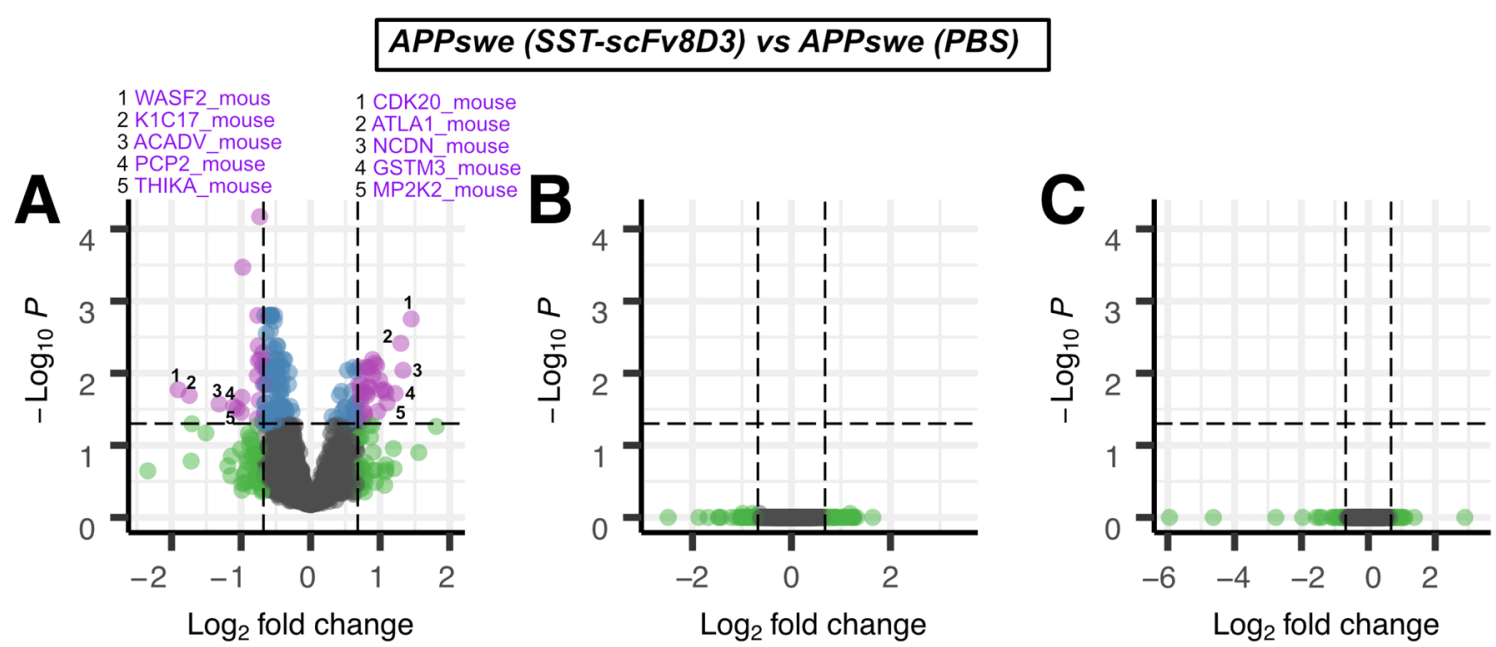

APPswe vs WT
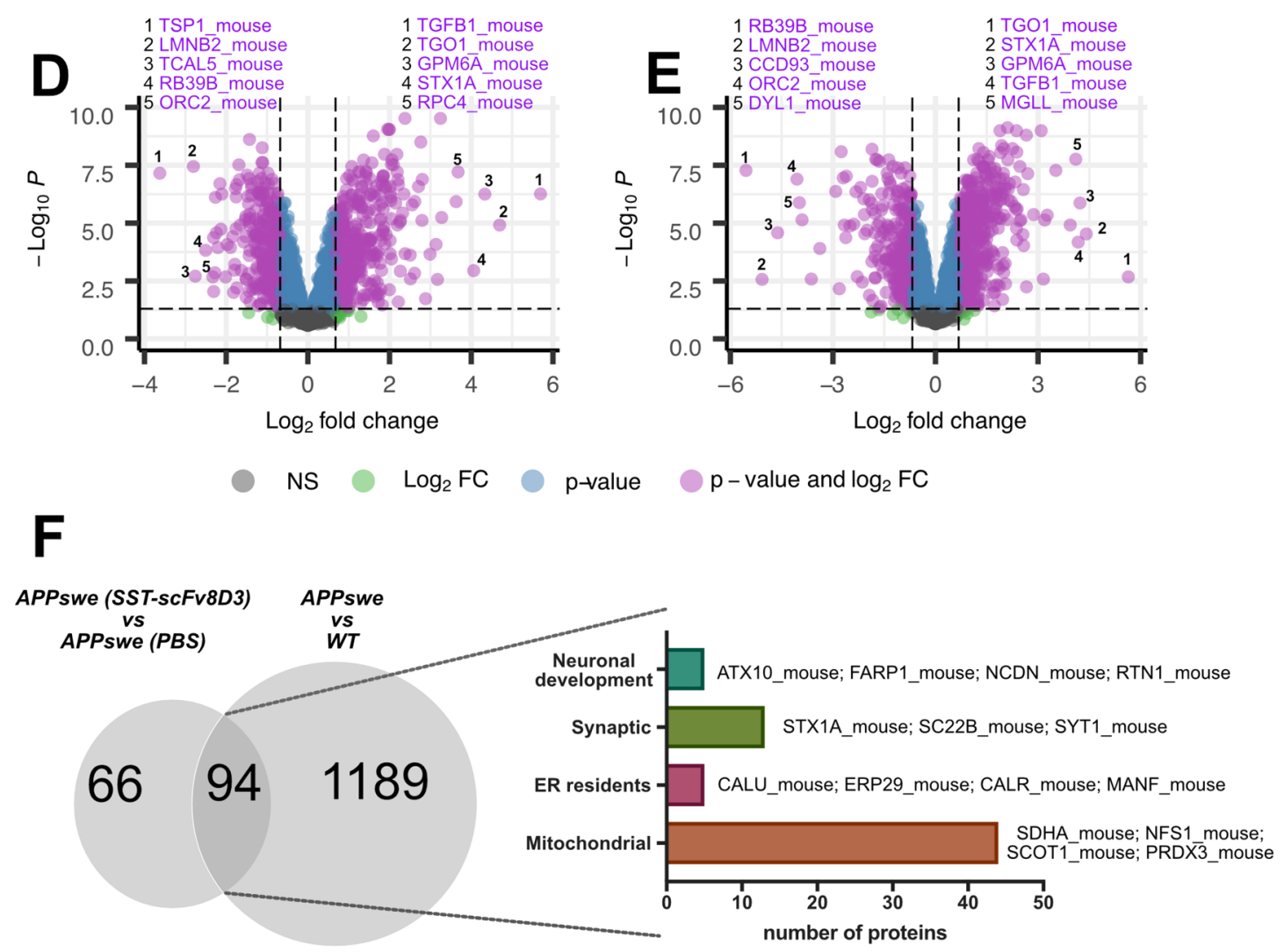

Figure 3. Summary of the LC-MS results. Volcano plots showing (A) hippocampal proteins with different levels between SST-scFv8D3 treated and PBS treated APPswe mice, (B and C) no significant alterations in protein levels between SST-scFv8D3 treated and PBS treated APPswe mice in the rest of cerebrum and cerebellum, (D) hippocampal proteins with different levels between APPswe and wild-type (WT) mice, and (E) proteins in the rest of cerebrum with different levels between APPswe and WT mice. Significantly affected proteins $(p<0.05)$ are displayed in blue and purple; not significantly (NS) affected proteins are presented in gray and green, whereas purple and green indicate a $\mid \log _{2}($ Fold change) $\mid>$ $\log _{2}(1.6)$. The top five significantly altered proteins based on fold change are highlighted in purple. (F) Overlap of all differentially expressed hippocampal proteins between SST-scFv8D3 treated and PBS treated APPswe mice with untreated APPswe and WT mice. Examples from the 94 proteins that overlapped between the two studies are presented. The majority of these proteins (44 out of 94 ) are mitochondrial.

APPswe mice compared to WT mice (Figure 3D,E). Proteins involved in autophagy such as the Ras-related proteins (Rab39) (RB39B_mouse) and the nuclear protein Lamin-B1 (LMNB1_mouse) represented some of the proteins with the most significantly decreased levels in the brain of APPswe mice compared to WT mice (Figure 3D,E).
Of the 160 differentially expressed proteins between SSTscFv8D3 and PBS treated APPswe, 94 proteins overlapped with the 1283 differentially expressed proteins between APPswe and WT mice in the hippocampus (Figure 3F and Table S5). Mitochondrial proteins represented the majority of these proteins (44 out of 94 ), followed by synaptic proteins, 
Table 1. Gene Ontology (GO) Enrichment Analysis Showing the Top 10 GO Terms for Biological Processes ${ }^{a}$

\begin{tabular}{|c|c|c|c|c|c|}
\hline GO biological process & detected & expected & fold enrichment & raw $p$-value & FDR \\
\hline fatty acid $\beta$-oxidation using acyl-CoA dehydrogenase & 6 & 0.57 & 10.55 & $1.80 \times 10^{-04}$ & $4.95 \times 10^{-02}$ \\
\hline tricarboxylic acid cycle & 14 & 1.54 & 9.07 & $2.44 \times 10^{-08}$ & $7.15 \times 10^{-05}$ \\
\hline branched-chain amino acid catabolic process & 7 & 0.81 & 8.62 & $1.21 \times 10^{-04}$ & $3.80 \times 10^{-02}$ \\
\hline branched-chain amino acid metabolic process & 7 & 0.81 & 8.62 & $1.21 \times 10^{-04}$ & $3.67 \times 10^{-02}$ \\
\hline acetyl-CoA metabolic process & 7 & 0.89 & 7.84 & $1.86 \times 10^{-04}$ & $4.95 \times 10^{-02}$ \\
\hline aerobic respiration & 15 & 1.95 & 7.70 & $3.72 \times 10^{-08}$ & $8.16 \times 10^{-05}$ \\
\hline cellular respiration & 18 & 2.52 & 7.15 & $3.44 \times 10^{-09}$ & $3.02 \times 10^{-05}$ \\
\hline purine nucleoside bisphosphate metabolic process & 12 & 1.87 & 6.42 & $3.93 \times 10^{-06}$ & $2.87 \times 10^{-03}$ \\
\hline ribonucleoside bisphosphate metabolic process & 12 & 1.87 & 6.42 & $3.93 \times 10^{-06}$ & $2.65 \times 10^{-03}$ \\
\hline nucleoside bisphosphate metabolic process & 12 & 1.87 & 6.42 & $3.93 \times 10^{-06}$ & $2.46 \times 10^{-03}$ \\
\hline
\end{tabular}

${ }^{a}$ Processes are of the significantly altered proteins in the hippocampal area of APPswe mice following SST-scFv8D3 treatment.

\section{Table 2. Gene Ontology Enrichment Analysis Showing the Top 10 GO Terms for Cellular Components ${ }^{a}$}

\begin{tabular}{|c|c|c|c|c|c|}
\hline GO cellular component & detected & expected & fold enrichment & raw $p$-value & FDR \\
\hline tricarboxylic acid cycle enzyme complex & 6 & 0.57 & 10.55 & $1.80 \times 10^{-04}$ & $1.66 \times 10^{-02}$ \\
\hline oxidoreductase complex & 10 & 1.46 & 6.84 & $1.74 \times 10^{-05}$ & $2.31 \times 10^{-03}$ \\
\hline mitochondrial matrix & 26 & 5.28 & 4.92 & $5.05 \times 10^{-10}$ & $3.02 \times 10^{-07}$ \\
\hline mitochondrial inner membrane & 17 & 4.87 & 3.49 & $3.10 \times 10^{-05}$ & $3.71 \times 10^{-03}$ \\
\hline organelle inner membrane & 17 & 5.04 & 3.38 & $4.42 \times 10^{-05}$ & $4.40 \times 10^{-03}$ \\
\hline mitochondrial membrane & 23 & 7.23 & 3.18 & $4.12 \times 10^{-06}$ & $6.15 \times 10^{-04}$ \\
\hline mitochondrial envelope & 26 & 8.20 & 3.17 & $9.43 \times 10^{-07}$ & $2.81 \times 10^{-04}$ \\
\hline envelope & 34 & 13.16 & 2.58 & $1.14 \times 10^{-06}$ & $2.72 \times 10^{-04}$ \\
\hline organelle envelope & 34 & 13.16 & 2.58 & $1.14 \times 10^{-06}$ & $2.27 \times 10^{-04}$ \\
\hline mitochondrion & 74 & 30.78 & 2.40 & $2.35 \times 10^{-13}$ & $2.80 \times 10^{-10}$ \\
\hline
\end{tabular}

${ }^{a}$ Components are of the significantly altered proteins in the hippocampal area of APPswe mice following SST-scFv8D3 treatment.

\section{A}

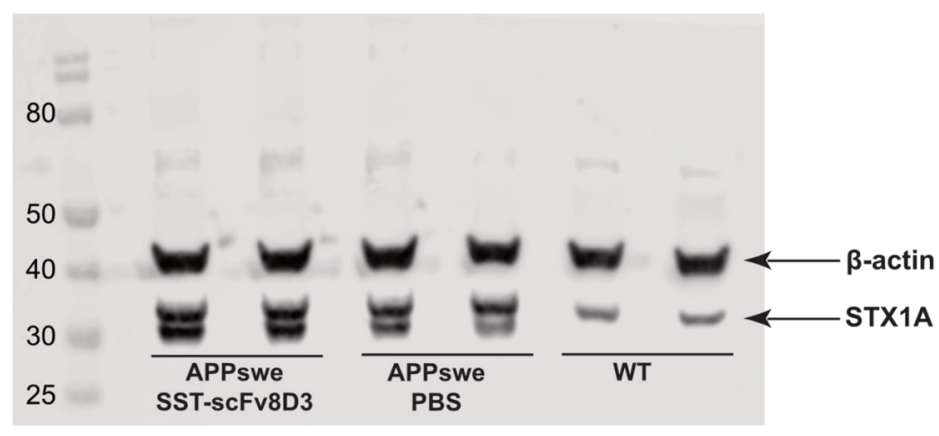

B

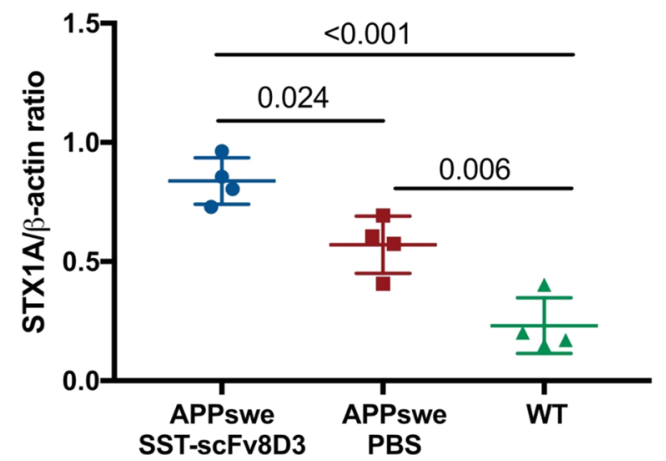

Figure 4. Western blot analysis of hippocampal lysates from SST-scFv8D3 treated APPswe mice $(n=4)$, PBS treated APPswe $(n=4)$, and WT controls $(n=4)$ for the detection and quantification of syntaxin-1A. (A) Representative Western blot image displaying qualitatively higher signals of syntaxin-1A in the SST-scFv8D3 treated APPswe lysates compared to those of PBS treated ones. Higher signals of syntaxin-1A in APPswe groups compared to those of WT controls. (B) Normalization against $\beta$-actin revealed a statistically significant increase in synatxin-1A in the hippocampal area of SST-scFv8D3 treated group compared to that of PBS treated APPswe mice. A statistically significant increase in synatxin-1A detected in the hippocampal area of APPswe mice compared to that of WT controls. Results are presented as mean \pm SD. One-way ANOVA followed by Bonferroni's posthoc test was applied to measure the presence of statistically significant differences in the results. A significant $p$-value is defined as $p<0.05$.

ER-resident proteins, and proteins involved in neuronal development (Figure 3F). Out of these 94 proteins, treatment with SST-scFv8D3 shifted the levels of 64 proteins toward WT levels (Table S5).

Syntaxin-1A Quantification. As mentioned in the previous section, synaptic proteins regulating trafficking across cell membranes represented most of the proteins with increased levels after treatment with SST-scFv8D3. Synaptic dysfunction is one of the main pathological events in Alzheimer's disease, where the interaction between $\mathrm{A} \beta$ and
syntaxin-1A has been demonstrated as a major contributing factor. ${ }^{16}$ The levels of the latter protein were increased in the hippocampal area of APPswe after treatment with SSTscFv8D3 measured with LC-MS (adjusted p-value 0.007) (Table S3). Moreover, syntaxin-1A was the fourth most significantly increased protein in the hippocampus of APPswe mice compared to WT controls (adjusted $p$-value $<0.0001$ ) (Table S4). To confirm these results, semiquantitative Western blot analysis was performed. Lysates from the hippocampal area of SST-scFv8D3 treated APPswe mice displayed a 

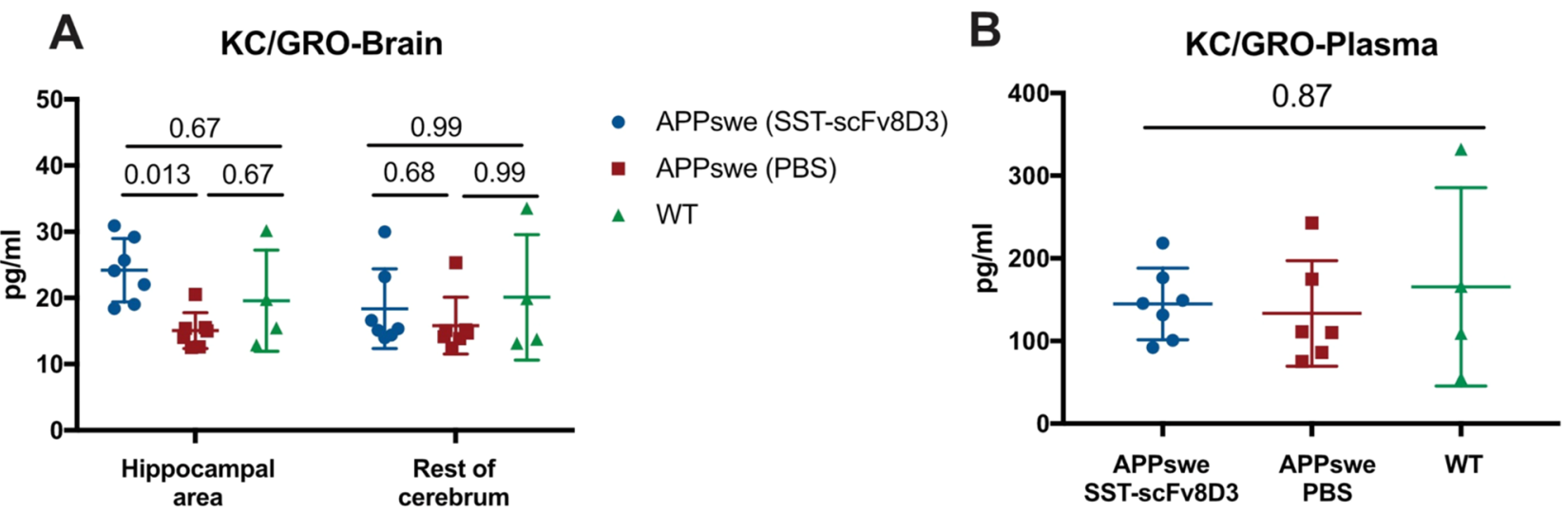

Figure 5. Concentration of $\mathrm{KC} / \mathrm{GRO}$ in the brain and blood of eight-month-old APPswe mice after triple intravenous injection of $1 \mathrm{mg} / \mathrm{kg}$ SSTscFv8D3 or PBS, using age-matched WT mice as controls. (A) A significant increase in hippocampal concentrations of KC/GRO in the SSTscFv8D3 treated group compared to those of PBS treated APPswe mice. No differences detected between the groups in the rest of the cerebrum ( $n$ $=7$ per group for APPswe mice) ( $n=4$ for WT mice). (B) No significant differences detected in plasma concentration of KC/GRO between SSTscFv8D3 treated APPswe $(n=7)$, PBS treated APPswe $(n=6)$, and WT mice $(n=4)$. Quantifications performed using a pro-inflammatory multiplex assay (MSD K125QTD). Results are presented as mean \pm SD. Kruskal-Wallis test followed by Dunn's post hoc analysis was applied to measure the presence of statistically significant differences in the results. A significant $p$-value is defined as $p<0.05$.

A

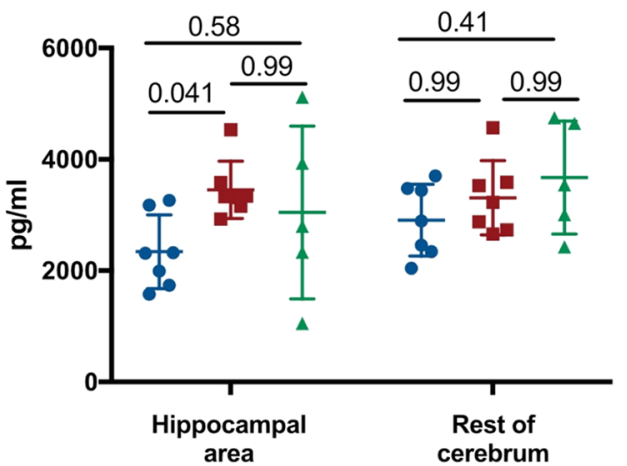

B

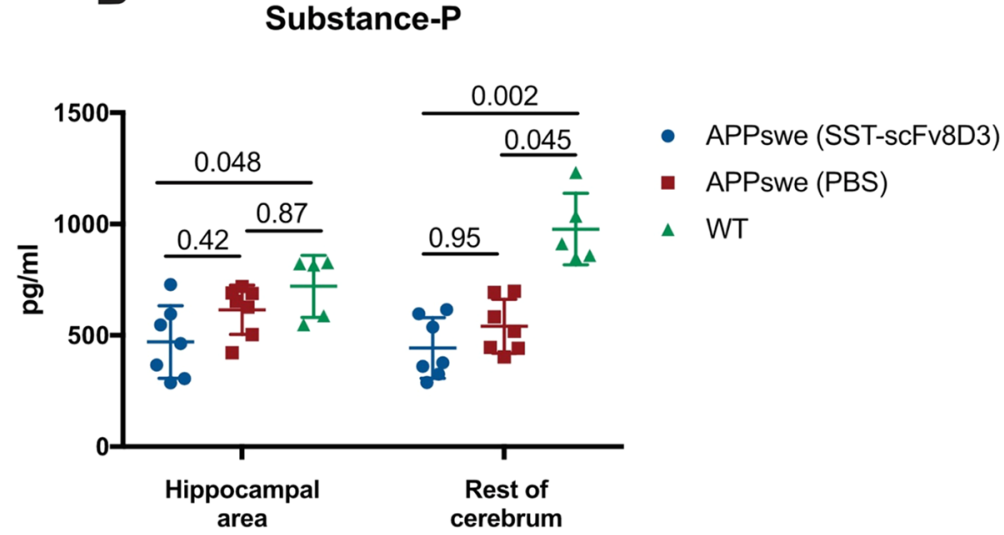

Figure 6. Brain concentrations of neuropeptide-Y and substance-P in eight-month-old APPswe mice after triple intravenous injection of $1 \mathrm{mg} / \mathrm{kg}$ SST-scFv8D3 or PBS, using age-matched WT mice as controls. (A) A significant decrease in hippocampal concentrations of neuropeptide-Y in the SST-scFv8D3 treated APPswe compared to those of the PBS treated APPswe mice. No differences detected between the groups in the rest of cerebrum. (B) No significant differences detected in the concentration of substance-P between SST-scFv8D3 treated and PBS treated APPswe mice. A significant decrease in substance-P concentration in APPswe mice compared to WT controls. Results are presented as mean \pm SD. Kruskal-Wallis test followed by Dunn's post hoc analysis was applied to measure the presence of statistically significant differences in the results. A significant $p$-value is defined as $p<0.05$. ( $n=7$ for APPswe treated with SST-scFv8D3; $n=7$ for APPswe treated with PBS; $n=5$ for WT).

significant increase in syntaxin-1A compared to that of the PBS treated group (Figure 4). In addition, APPswe mice displayed a significant increase in syntaxin-1A compared to that of the WT controls (Figure 4), further confirming the LC-MS results.

KC/GRO Chemokine Quantification. In our previous study, no significant differences were detected in the concentration of interleukins (IL-1b, IL-5, IL-6, and IL-12) after treatment with SST-scFv8D3. ${ }^{6}$ However, the response of the immune system to such treatment could include changes in the levels of inflammatory markers other than interleukins. In the current study, we wanted to study immune cell activation and migration. For this purpose, we quantified the concentration of growth-regulated $\alpha(\mathrm{KC} / \mathrm{GRO})$, a chemokine that regulates the activation of neutrophils and macrophages. ${ }^{17}$ This chemokine was not detected in our LC-MS analysis. Using the MSD multiplex assay, a significant increase in the concentration of $\mathrm{KC} / \mathrm{GRO}$ was detected in the hippocampal area of SST-scFv8D3 treated APPswe mice compared to that of the PBS treated group (Figure 5A). No differences were detected in the rest of the cerebrum (Figure 5A). The increased concentrations of $\mathrm{KC} / \mathrm{GRO}$ were hippocampalspecific, as no differences were detected in the concentration of this chemokine in the blood (Figure 5B).

Neuropeptide-Y and Substance-P Quantification. We have previously demonstrated the ability of the bispecific SSTscFv8D3 protein drug to degrade hippocampal $\mathrm{A} \beta 42$ as a result of enhanced neprilysin activation. ${ }^{6}$ In addition to $\mathrm{A} \beta$, neprilysin degrades other peptides in the brain, including neuropeptide-Y and tachykinins such as substance-P. ${ }^{18}$ In the current study, we aimed at quantifying the brain concentration of other neprilysin substrates, focusing on neuropeptide-Y and substance-P. A significant decrease in the concentration of neuropeptide- $\mathrm{Y}$ was detected only in the hippocampal area of the SST-scFv8D3 treated group (Figure 6A), the area where neprilysin activity was selectively enhanced. ${ }^{6}$ No differences were detected in the brain concentration of substance-P 
between SST-scFv8D3 treated and PBS treated APPswe mice (Figure 6B). APPswe mice demonstrated a significant decrease in substance-P concentration compared to that of the WT controls (Figure 6B).

\section{- DISCUSSION}

In this study, we demonstrate the ability of the SST peptide with a protein-based BBB transporter (SST-scFv8D3) to regulate the levels of several proteins in the hippocampus of the APPswe transgenic mouse model of Alzheimer's disease. The effects were selectively targeted to the hippocampal area of the brain, where SST receptors are highly expressed. We have previously displayed the ability of SST-scFv8D3 to enter the brain parenchyma and to localize predominately in the hippocampus and its surrounding cortical area, selectively enhancing neprilysin expression and reducing the $\mathrm{A} \beta 42$ concentration in this brain region. ${ }^{6}$ No effects of the formed fusion protein on the proteome of the cerebellum or rest of the cerebrum were observed.

The altered protein levels measured in this study after treatment with SST-scFv8D3 can be attributed to several possible reasons. The removal of excess $\mathrm{A} \beta$ peptides could be followed by the alteration in hippocampal protein levels detected in the SST-scFv8D3 treated group. However, the observed changes could also be related to SST receptor activation that is associated with numerous downstream events unrelated to neprilysin activation. Furthermore, neprilysinmediated degradation of substrates other than $\mathrm{A} \beta$, and the interaction of SST with other proteins, could also contribute to the detected effects in this study.

Because our protein design also includes a BBB transporter, it is possible that some of the effects are mediated by this part of the construct rather than the SST. The scFv8D3 transporter binds to TfR and induces endocytosis of this complex. TfR is abundantly expressed on the endothelial cells of the BBB but not on other cell types in the brain. ${ }^{19}$ The analyzed brain regions in this study contain a small proportion of BBB cells, resulting in a possible alteration of protein levels related to the enhanced endocytosis or other downstream effects due to the interaction with TfR. Nevertheless, we have previously demonstrated an even brain distribution of the BBB transporter, which is not limited to a specific region. ${ }^{14}$ However, the SST-scFv8D3 construct has a longer retention time in the hippocampal area, where the SST receptors are highly expressed, ${ }^{6}$ indicating that TfR binding is not as essential for half-life extension as the interaction with SST receptors. Because the observed changes in protein levels were exclusive to the hippocampal area (Figure 3A), SST-scFv8D3 interaction with $\mathrm{TfR}$ is only likely to contribute to a small proportion of the detected alterations in protein levels after treatment. Having this in mind, the following sections are based on discussing the altered protein levels only in regard to Alzheimer's disease:

Proteins with the Most Significant Decrease (Based on Fold Change) after SST-scFv8D3 Treatment. The top 10 proteins whose levels were significantly decreased after treatment with SST-scFv8D3 were mainly mitochondrial and endoplasmic reticulum (ER) residents (6 out of 10), which are further described in the following sections. Two plasma membrane proteins were also detected among the top 10 downregulated proteins after SST-scFv8D3 treatment. These included Wiskott-Aldrich syndrome protein family members 2 (highest significant decrease in terms of fold-change after treatment with SST-scFv8D3), which is involved in the processes of protein folding and Rho GTPase-activating protein 35 , which is involved in cell differentiation. In addition, the two extracellular proteins Clathrin light chain B and Purkinje cell protein 2 represented the rest of the top 10 proteins with the most significant decrease. The latter protein, Purkinje cell protein 2, is involved in modulating cell-signaling processes. In the following sections, we discuss interesting leads from the downregulated proteins in relation to their involvement in Alzheimer's disease pathology.

Mitochondrial Proteins. In our treatment study, around half of the proteins whose levels were significantly decreased in the SST-scFv8D3 treated group were mitochondrial, involved mainly in processes of fatty acid oxidation, amino acid metabolism, energy production, autophagy, and protein assembly. Mitochondrial dysfunction is one of the key pathological events contributing to the onset and progression of Alzheimer's disease. ${ }^{20}$ Several studies have demonstrated the ability of aggregated $\mathrm{A} \beta$ to accumulate and interact with mitochondrial proteins, both in transgenic mouse models overexpressing $\mathrm{A} \beta$ and in Alzheimer's disease patients' brains. $^{21,22}$ Transgenic Alzheimer's disease mice harboring the Swedish mutation exhibited mitochondrial accumulation of A $\beta 40$ and 42 isoforms at early stages of the disease before

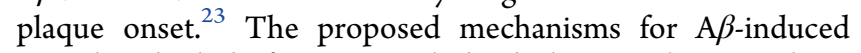
mitochondrial dysfunction include decline in the mitochondrial import of physiologically relevant proteins, increased reactive-oxygen species (ROS), and decreased mitochondrial membrane potential. ${ }^{24}$ To compensate for such effects, several mitochondrial proteins are upregulated in the early stages of the disease. This upregulation could be attributed to proteins that propagate the disease or proteins that contribute to a compensatory mechanism that protects the mice from the overexpression of $\mathrm{A} \beta$ and the subsequent downstream effects. Examples include thioredoxin-reductase and citrate synthase that have been previously shown to be upregulated in Alzheimer's disease brains ${ }^{25}$ and in an 8-month-old transgenic mouse model of Alzheimer's disease, ${ }^{26}$ respectively. These two mitochondrial proteins among several others were downregulated in the hippocampus of the study group, which could be a sign of an altered mitochondrial function resulting from enhanced degradation of $\mathrm{A} \beta 42$ by SST-scFv8D3. In order to assess whether the effects of SST-scFv8D3 treatment reestablishes normal hippocampal levels of the mitochondrial proteins, we performed a transgenic study including agematched APPswe mice and WT controls. Interestingly, $80 \%$ of the mitochondrial proteins that were downregulated after SSTscFv8D3 treatment displayed significantly higher levels in the hippocampi of APPswe mice compared to those of WT mice, strongly suggesting that SST-scFv8D3 corrects these altered mitochondrial proteins toward WT levels. The data obtained in the transgenic study are also consistent with previously published findings, which show a significant increase in the levels of mitochondrial proteins including stress-70 protein, 10 $\mathrm{kDa}$ heat shock protein, superoxide dismutase, fumarate hydratase, aspartate aminotransferase, and pyruvate dehydrogenase in the brains of neprilysin knockout APPswe mice compared to those of neprilysin knockout WT mice. ${ }^{25}$ In our study, all these mentioned proteins are downregulated in the hippocampi of APPswe mice treated with SST-scFv8D3, an effect that could be mediated by the enhanced degradation of A $\beta 42$. These results could indicate that the observed increase in the levels of the mitochondrial proteins was independent of 
neprilysin levels and instead was due to $\mathrm{A} \beta$ overproduction and aggregation. However, SST peptide has displayed the ability of binding and interacting with several mitochondrial proteins, including aldehyde dehydrogenase in brain homogenates, ${ }^{27}$ which was also downregulated in our study. Moreover, neprilysin inhibitors used in the treatment of heart failure has been associated with an increase in the levels of mitochondrial proteins, ${ }^{28}$ several of which are downregulated in our study where neprilysin levels are enhanced. ${ }^{6}$ These studies might suggest that the regulation of mitochondrial proteins is not exclusively mediated by decreased $\mathrm{A} \beta$ levels but can also be mediated by SST interaction with other proteins and neprilysin overexpression.

Endoplasmic Reticulum Proteins. In addition to mitochondria, several of the proteins with significantly lower levels in the study group are ER residents. The expression and function of these proteins in Alzheimer's disease are not well-studied. However, the ER can provide a suitable oxidizing environment for the processing of APP and generation of $\mathrm{A} \beta$ peptides, where ER-resident proteins such as calreticulin play a role. ${ }^{29}$ In the current study, the levels of calreticulin as well as other ER proteins such as calumenin and endoplasmic reticulum resident protein 29 were significantly decreased in the hippocampi of APPswe mice compared to those of the WT controls. Treatment of APPswe mice with SST-scFv8D3 decreased their levels even more, an effect that needs further investigation.

Amyloid-Like Protein 1 (APLP-1). Importantly, treatment with SST-scFv8D3 was associated with a $40 \%$ decrease in the hippocampal levels of amyloid-like protein 1 (APLP-1). No studies have demonstrated the ability of neprilysin to degrade APLP-1. However, interaction between the latter protein and neprilysin promoter has been previously reported. ${ }^{30}$ The involvement of APLP-1 in Alzheimer's disease has been extensively discussed. APLP-1 is a member of the amyloid precursor family and is highly homologous to APP, showing similar enzymatic digestion processing into short $\mathrm{A} \beta$-like peptides that accumulate in Alzheimer's disease brains. ${ }^{31,32}$ Moreover, APLP-1 has been suggested as a cerebrospinal fluid (CSF) biomarker in Alzheimer's disease. ${ }^{33}$ Decreased levels of this protein in the hippocampal area might further support the anti-Alzheimer's effects of SST-scFv8D3.

Neuropeptide- $Y$ and Substance-P. We have previously demonstrated a significant decrease in the concentration of A $\beta 42$ in the hippocampi of APPswe mice after SST-scFv8D3 treatment. $^{6}$ This is an effect that was mediated by the significant and selective increase in hippocampal neprilysin activity. ${ }^{6}$ However, neprilysin is a promiscuous enzyme capable of degrading a variety of substrates in the brain other than $\mathrm{A} \beta{ }^{18}$ For this reason, we quantified the brain concentrations of neuropeptide-Y and substance-P, two peptide substrates of neprilysin that are highly expressed in the brain. Treatment with SST-scFv8D3 was associated with a significant decrease in neuropeptide- $Y$ concentration, but not substance-P, selectively in the hippocampal area (Figure 6). Enhanced degradation of neuropeptide- $Y$ in the hippocampus could further demonstrate the increased activity and expression of neprilysin in this specific brain region after SST-scFv8D3 treatment. However, the enhanced degradation of neuropeptide-Y might be associated with possible adverse effects, because the peptide is involved in regulating several relevant physiological processes in the brain such as food intake and energy homeostasis. Of note, APPswe mice demonstrated a significant decrease in substance-P concentration compared to that of WT controls, an effect that was even more evident in areas outside the hippocampus as demonstrated previously (Figure 6). ${ }^{34}$

Proteins with the Most Significant Increase after SSTscFv8D3 Treatment. Proteins regulating trafficking across the cell membrane represented around $40 \%$ of the proteins with significantly higher levels found in the hippocampal area of SST-scFv8D3 treated APPswe mice. Proteins involved in processes of axon and dendrite assembly, synapse formation, cell-to-cell adhesion, and ubiquitination represented the rest of the upregulated proteins. In the following sections, we discussed some of these proteins that are involved in Alzheimer's disease pathology.

$K C / G R O$ Chemokine. Inflammation plays a major role in the pathology of Alzheimer's disease. ${ }^{15}$ We have previously demonstrated no significant alteration in the concentrations of interleukins (IL-1b, IL-5, IL-6, and IL12) in the brains of APPswe mice after treatment with SST-scFv8D3. ${ }^{6}$ However, this is not a complete list of cytokines, and the immune system might respond to treatments in several ways that do not include alterations in interleukin concentrations. To further study immune cell-activation, we quantified the concentration of $\mathrm{KC} / \mathrm{GRO}$, a chemokine expressed in endothelial cells and macrophages. This chemokine plays an integral role in the activation and migration of immune cells like neutrophils. ${ }^{17}$ Its receptor, CXCR2, is expressed by neurons and microglia. ${ }^{35}$ Treatment with SST-scFv8D3 was associated with a significant and selective increase in $\mathrm{KC} / \mathrm{GRO}$ concentration in the hippocampal area (Figure 5A). No changes were detected in the rest of the cerebrum, nor in the blood, indicating that the increase in $\mathrm{KC} / \mathrm{GRO}$ concentration is correlated with SSTscFv8D3 treatment effects. Downstream effects of increased KC/GRO concentration by SST-scFv8D3 treatment could contribute to pro-inflammatory microglial activation mediated by the interaction of $\mathrm{KC} / \mathrm{GRO}$ with its receptor. ${ }^{36}$ In addition, enhanced expression of $\mathrm{KC} / \mathrm{GRO}$ can be associated with infiltration of immune cells from the periphery into the brain, including both monocytes ${ }^{37}$ and neutrophils, ${ }^{38}$ aiding the phagocytic removal of degraded $\mathrm{A} \beta$. Furthermore, our results could hint to the important effects that $\mathrm{KC} / \mathrm{GRO}$ chemokine might exert in the early stages of the disease.

Syntaxin-1A. An interesting protein that was upregulated in the hippocampus of SST-scFv8D3 treated APPswe mice was the SNARE (soluble $N$-ethylmaleimide-sensitive fusion protein attachment protein receptor) protein syntaxin-1A. The enhanced levels of this protein in the SST-scFv8D3 treated group were also confirmed with Western blot (Figure 4). Furthermore, syntaxin-1A was among the most significantly upregulated proteins in the brain of APPswe mice compared to WT controls (Figure 3D,E). Although previous studies have demonstrated reduced syntaxin-1A levels in Alzheimer's disease brains, ${ }^{39}$ the observed increase in the levels of this protein in our transgenic mouse model can be a compensatory mechanism to the overexpression of APP in the brain of these mice. Importantly, $\mathrm{A} \beta$ oligomers have shown the ability of binding to syntaxin-1A, inhibiting SNARE complex formation and subsequently reducing SNARE-mediated vesicle fusion. ${ }^{16}$ Treatment of APPswe mice with SST-scFv8D3 further increased syntaxin-1A levels (Figures $3 \mathrm{~A}$ and 4 ). This can be attributed to the role of SST in regulating the presynaptic localization of neprilysin, which has been suggested to be partially mediated by SNARE proteins, including syntaxin-1A. ${ }^{8}$ 
Other SNARE-Related Proteins. In addition to syntaxin-1A, the levels of other SNARE-related proteins such as synaptotagmin-1 and synaptobrevin homologue YKT6 were also increased in the hippocampi of SST-scFv8D3 treated mice. Although no interaction between $\mathrm{A} \beta$ and these proteins has been reported, their involvement in Alzheimer's disease has been previously discussed. Synaptotagmin has been shown to have the ability to promote $\mathrm{A} \beta$ generation. ${ }^{40}$ In addition, our current transgenic study further demonstrated a significant increase in hippocampal levels of synaptotagmin in APPswe mice compared to those of WT controls. However, knockdown of neprilysin in airway epithelial cells has been associated with a significant decrease in synaptotagmin gene expression. ${ }^{41}$ With that said, it is rather likely that the increased synaptotagmin levels we see in the hippocampi are due to the increase in the neprilysin concentration.

Neuronal Growth Proteins. Treatment with SST-scFv8D3 displayed a significant increase in the levels of proteins involved in neuronal growth and development. Examples include serine/threonine protein kinase BRSK2 (highest significant increase in terms of fold-change after treatment with SST-scFv8D3), atlastin-1 (second highest significant increase after treatment), and ataxin-10. The latter protein has recently been suggested to be related to Alzheimer's disease, as $\mathrm{A} \beta$ accumulation and neuronal degeneration were observed in the hippocampus when ataxin-1 is knocked out in a transgenic mouse model of Alzheimer's disease. ${ }^{42}$ Serine/ threonine protein phosphatase 2A (gamma and epsilon isoforms) was also among the upregulated proteins in the treatment group. Overproduction and accumulation of $\mathrm{A} \beta$ caused a strong downregulation of the activity of this enzyme in the brain during Alzheimer's disease. ${ }^{43,44}$ In addition, coimmunoprecipitation studies showed the interaction between neprilysin and serine phosphatase, as well as treatment of primary cultures with serine-phosphatase inhibitors, significantly reduced cell-surface neprilysin activity. ${ }^{45}$ Therefore, enhanced neprilysin activity and reduced $\mathrm{A} \beta$ accumulation by our SST-scFv8D3 could probably explain the observed upregulated levels of this enzyme. Finally, another interesting protein group whose levels are significantly increased after treatment is the reticulons (reticulons 1, 3, and 4). In addition to their role in mediating trafficking across the cell membrane, they are involved in neuronal and glial cell growth. They have been involved in modulating the activity of $\beta$-secretase, the enzyme involved in the proteolytic digestion of amyloid-precursor protein (APP) into A $\beta$. Enhancing the expression levels of these proteins has been associated with reduced accumulation of $\mathrm{A} \beta{ }^{46}$ Importantly, our current study demonstrated a significant decrease in the levels of neuronal growth proteins (ataxin-10, neurochondrin, and reticulon-1, among others) in the hippocampi of APPswe mice compared to those of WT controls, further suggesting that SST-scFv8D3 treatment shifts these altered proteins toward normal levels.

Dynamin-1. It is worth mentioning that treatment with SST-scFv8D3 was associated with increased levels of the $\mathrm{A} \beta$ aggregation regulating the protein dynamin-1. This protein is extensively studied in Alzheimer's disease, where it has been demonstrated to be involved in regulating $\mathrm{A} \beta$ formation and aiding its aggregation. ${ }^{47,48}$ Moreover, APPswe mice with neprilysin knockout displayed a significant increase in dynamin-1 levels. ${ }^{49}$ Whether such an unexpected increase is mediated by our $\mathrm{A} \beta$ degrading SST-scFv8D3 protein drug or it is secondary to other interaction partners of SST needs to be further investigated. Importantly, despite its role in enhancing A $\beta$ formation, dynamin- 1 has demonstrated some neuroprotective role in synapse development in the central nervous system. ${ }^{47}$ Of note, dynamin-1 is involved in mediating endocytosis and synapse development. ${ }^{50}$ Therefore, the increased hippocampal levels of this protein after SSTscFv8D3 treatment might indicate enhanced endocytosis due to scFv8D3 interaction with TfR and/or SST interaction with SST receptors.

Dual-Specificity Mitogen Activated Protein Kinases. Another important protein group with significantly increased levels in the treated APPswe mice were the dual-specificity mitogen activated protein kinases (subtype 2 and 1 representing the fifth and sixth most significantly upregulated proteins after SST-scFv8D3 treatment, respectively). These proteins are an integral part of the mitogen-activated protein (MAP) signaling cascade, involved in regulating immune responses and inflammation and promoting cell survival. ${ }^{51}$ They have been suggested as neuroprotective proteins in several brain diseases through their ability to dephosphorylate important MAP-kinases. ${ }^{52}$ In addition, dephosphorylation of APP by these enzymes has been shown to be associated with the decreased ability of $\beta$-secretase to cleave APP and produce $\mathrm{A} \beta .^{53}$ The significant decrease in the levels of these proteins in the brains of APPswe mice compared to those of WT controls and their upregulation after SST-scFv8D3 treatment might further support the therapeutic significance of our proteindrug.

Conclusion. We have shown that treatment with SSTscFv8D3 generated multiple anti-Alzheimer's disease effects. Identification of proteins, such as syntaxin-1A and the chemokine $\mathrm{KC} / \mathrm{GRO}$, highlights their potential role in Alzheimer's disease, opening up new possibilities to further study their association with $\mathrm{A} \beta$ pathology. We also saw that mitochondrial and neuronal developmental proteins that are frequently altered in Alzheimer's disease were shifted by the SST-scFv8D3 treatment towards normal levels. Our findings suggest that the somatostatin peptide not only lowers the amount of $\mathrm{A} \beta$ in the hippocampus, as we have shown earlier, ${ }^{6}$ but is also associated with other previously undiscovered beneficial effects. As these effects are closely related to cognition, we believe that the results of this study have strengthened the continued evaluation of the somatostatin peptide as a therapeutic option to ameliorate the cognitive dysfunction presented in Alzheimer's disease.

\section{MATERIALS AND METHODS}

Animals. Female and male 8-month-old APPswe transgenic mice, harboring the Swedish mutation (A $\beta$ PP KM670/671NL), were used in this study. Littermates were used as wild-type controls. The mice were housed in an animal facility at Uppsala University, with free access to water and food and in rooms with controlled temperature and humidity. Experimental procedures were approved by the Uppsala County Animal Ethics Board (\#5.8.18-13350/17).

SST-scFv8D3 Treatment. APPswe mice were divided into two groups ( $n=7$ per group). Using the tail vein, $1 \mathrm{mg} / \mathrm{kg} \mathrm{SST-scFv8D3,}$ recombinantly produced as described previously, was intravenously injected into the study group every $36 \mathrm{~h}$ (three injections in total). SST-scFv8D3 is a fusion protein consisting of the 14 amino acid SST peptide (AGCKNFFWKTFTSC) linked to scFv8D3 with an in-house designed linker (APGSYTGSAPG). The second group received an intravenous injection of phosphate buffered saline (PBS) at the same time points as the study group. At $24 \mathrm{~h}$ after the last injection, mice were euthanized by transcardial perfusion with $0.9 \%$ physiological 
saline. Brains were isolated, and three brain regions (hippocampal area, rest of cerebrum, and cerebellum) were dissected on ice and stored in $-80{ }^{\circ} \mathrm{C}$. The three brain regions were homogenized at a $1: 5$ weight/volume ratio with the homogenization buffer $(20 \mathrm{mM}$ Tris, $137 \mathrm{mM} \mathrm{NaCl}, \mathrm{pH}$ 7.6) containing $1 \%$ (volume/volume) protease inhibitor (Thermo Scientific, Waltham, MA, USA). The same procedures regarding mice euthanization and tissue processing were applied for the transgenic study, including untreated APPswe mice and WT controls.

Tryptic Digestion. The homogenates of the three brain regions were lysed using tip sonication at $4{ }^{\circ} \mathrm{C}$ (pulse $10 \times 1 \mathrm{~s}$, rest $1 \mathrm{~s}$, amplitude 30\%; Vibra cell ultrasonic processor with $3 \mathrm{~mm}$ probe; Sonics, Newtown, CT, USA) in a buffer containing 20 mM HEPES, 6 $\mathrm{M}$ urea, $1 \mu \mathrm{g} / \mathrm{mL}$ RapiGest SF Surfactant (Waters Corporation, Milford, MA, USA), and 1\% (volume/volume) protease inhibitor (Thermo Scientific, Waltham, MA, USA). Total protein $(20 \mu \mathrm{g})$ was transferred onto centrifugal filter units (Microcon-30 kDa; Merck, Darmstadt, Germany) for protein purification and tryptic digestion according to a Filter Aided Sample Preparation (FASP) protocol, ${ }^{54}$ with minor modifications. Briefly, the samples were washed with a buffer composed of $8 \mathrm{M}$ urea and $100 \mathrm{mM}$ Tris ( $\mathrm{pH} 8.5$ ). After the reduction with $8 \mathrm{mM}$ dithiothreitol, the alkylation with $50 \mathrm{mM}$ iodoacetamide and the removal of excess iodoacetamide with $8 \mathrm{mM}$ dithiothreitol this washing step was repeated each time. Before adding trypsin (enzyme-to-protein ratio 1:50 (weight/weight)), the filter was washed with $\mathrm{NH}_{4} \mathrm{HCO}_{3}$ three times. After $16 \mathrm{~h}$ of incubation in a wet chamber at $37^{\circ} \mathrm{C}$, the peptides were washed from the filter with 50 $\mathrm{mM} \mathrm{NH} \mathrm{NHCO}_{3}$. Trifluoroacetic acid was added to create a final concentration of $1 \%$ (volume/volume). The samples were dried down at $45{ }^{\circ} \mathrm{C}$ and afterwards reconstituted in $3 \%$ acetonitrile and $0.1 \%$ formic acid in water to a final protein concentration of $150 \mathrm{ng} / \mu \mathrm{L}$.

Liquid Chromatography-Mass Spectrometry (LC-MS). The tryptic peptides were analyzed on a nanoAcquity UPLC system coupled to a Synapt G2-Si HDMS mass spectrometer equipped with an electrospray ionization source (Waters Corporation, Manchester, UK). Mobile phase A contained $0.1 \%$ formic acid and 3\% dimethyl sulfoxide in water and mobile phase B $0.1 \%$ formic acid and $3 \%$ dimethyl sulfoxide in acetonitrile. Protein $(300 \mathrm{ng})$ was injected in trapping mode on a $\mathrm{C} 18,5 \mu \mathrm{m}, 180 \mu \mathrm{m} \times 20 \mathrm{~mm}$ trap column (Waters Corporation). The following HSS-T3 C18 $1.8 \mu \mathrm{m}, 75 \mu \mathrm{m} \times$ $250 \mathrm{~mm}$ analytical column (Waters Corporation) was kept at $40{ }^{\circ} \mathrm{C}$. For peptide separation, a gradient was run from $3-40 \%$ (volume/ volume) mobile phase $\mathrm{B}$ over $120 \mathrm{~min}$ at a flow rate of $0.3 \mu \mathrm{L} / \mathrm{min}$. The peptides were analyzed in positive ionization mode by using the UDMS $^{\mathrm{E}}$ approach published previously. ${ }^{54,55}$ A lock mass solution of [Glu1]-fibrinopeptide $\mathrm{B}(0.1 \mu \mathrm{M})$ and leu-enkephalin $(1 \mu \mathrm{M})$ was introduced to the mass spectrometer via the reference sprayer every $60 \mathrm{~s}$. The reproducibility and stability of the method was controlled with ten HeLa digest control samples (Thermo Scientific, Waltham, MA, USA) that were analyzed interspersed between the brain samples. A schematic illustration of the work flow is presented in Figure 2.

LC-MS Data Processing and Label-Free Quantification Analysis. Raw data was processed using ProteinLynx Global Server (version 3.0.3, Waters Corporation, Milford, MA, USA). The database search against a randomized UniProt mouse database (UniProtKB version $14 / 01 / 2020$ ) was done with a false discovery rate (FDR) of 0.01 . Trypsin was set as the digest reagent. Carbamidomethyl cysteine was set as the fixed modification. Acetyl lysine, C-terminal amidation, asparagine deamidation, glutamine deamidation, and methionine oxidation were set as variable modifications. One missed cleavage per peptide was allowed. Minimum fragment ion matches per peptide were 1 and per protein were 3 . Minimum peptide matches per protein were 2.

ISOQuant 1.8 was used for label-free quantification analysis. ${ }^{54,55}$ The workflow included nonlinear retention time alignment, signal clustering based on accurate mass, retention and drift time, annotation of signal clusters using PLGS identifications, intensity normalization, and protein isoform and homology filtering. The average intensity of the three most intense peptides of each protein was used for relative protein quantification (TOP3 quantification). The software settings are described in Table $\mathrm{S} 6$.

Results from ISOQuant were further analyzed for changes in protein levels in R 3.6.3 with the empirical Bayes method using the package limma. ${ }^{56}$ Histogram plots of the raw $p$-values can be found in Figure S2. Obtained $p$-values were adjusted for multiple comparisons with the package $q$-value, setting the FDR $<0.05 .^{57}$ All $\log _{2}$-fold changes with an adjusted $p$-value $<0.05$ were considered statistically significant. Please note that all figures and text in the results and discussion refer to adjusted $p$-values.

Altered protein levels were processed for GO enrichment analysis with Panther 15.0 using all identified proteins as reference.

Western Blot Analysis. APPswe lysates from both SST-scFv8D3 treated and PBS treated groups, as well as WT controls, were mixed with LDS sample buffer (Thermo Scientific, Waltham, MA, USA) and Bolt sample reducing agent (Thermo Scientific, Waltham, MA, USA) and loaded onto Bolt 4-12\% Bis-tris plus gels (Thermo Scientific, Waltham, MA, USA). Gels were run at $80 \mathrm{~V}$ for $1-2 \mathrm{~h}$ followed by transfer onto PVDF-membranes (Merck, Darmstadt, Germany). Membranes were blocked with 5\% dry milk in TBS-Tween for $1 \mathrm{~h}$ at RT, followed by overnight staining at $4{ }^{\circ} \mathrm{C}$ with primary antibodies anti-syntaxin antibody (ab41453, Abcam, Cambridge, United Kingdom) and anti- $\beta$-actin antibody, clone AC-15 (Sigma-Aldrich, Stockholm, Sweden). The following day, membranes were washed with TBS-Tween buffer and incubated with goat antimouse IgG Alexa 680 (A21057, Life Technologies, Waltham, MA, USA) and donkey antirabbit IgG Alexa 800 (A32808, Invitrogen, Waltham, MA, USA) secondary antibodies for $1 \mathrm{~h}$ at RT. After they were washed three times with TBS-Tween buffer, signals were developed using the LICOR odyssey machine (LI-COR biosciences, Homburg, Germany). Signal intensity was measured using Image Studio software (version 5.2.5). The measured signals from syntaxin were normalized against the loading control ( $\beta$-actin).

KC/GRO Quantification. Concentration of KC/GRO in the brain and plasma of SST-scFv8D3 treated and PBS treated APPswe mice, as well as WT controls, was quantified using a pro-inflammatory cytokine multiplex assay (K152QTD, Meso Scale Diagnostics, Rockville, MD, USA), according to the manufacturer protocol. Lysates from hippocampal area and rest of cerebrum were diluted at the $1: 1$ volume/volume ratio, while plasma samples were diluted at the $1: 10$ volume/volume ratio.

Neuropeptide-Y and Substance-P Quantification. Brain concentrations of neuropeptide- $Y$ and substance-P were quantified in treated APPswe mice as well as WT controls using ELISA, according to the manufacturer protocol (A3330/A80235, Antibodies. com, Cambridge, United Kingdom). Lysates from the hippocampal area and the rest of the cerebrum were diluted at the 1:5 volume/ volume ratio.

Statistical Analyses. Non-LC-MS results are presented as mean \pm SD. The Shapiro-Wilk test was applied to test for normality. Data that passed the normality test were analyzed with one-way ANOVA followed by Bonferroni's posthoc test. Data that did not pass the normality test were analyzed with the Kruskal-Wallis test followed by Dunn's post hoc analysis. A significant $p$-value is defined as $p<0.05$.

\section{ASSOCIATED CONTENT}

\section{Supporting Information}

The Supporting Information is available free of charge at https://pubs.acs.org/doi/10.1021/acschemneuro.1c00303.

List of 1869 proteins quantified across all 42 samples, list of proteins with lower levels in hippocampus, list of proteins with higher levels in hippocampus, list of 2487 proteins quantified across all samples in hippocampus and rest of cerebrum, list of 94 hippocampal proteins that overlapped between 160 differentially expressed proteins in treatment study and 1283 differentially expressed proteins in transgenic study, ISOQuant 1.8 software settings used for label-free quantification 
analysis of LC-MS data, heatmap of differentially expressed hippocampal proteins, and histograms of raw $p$-values (PDF)

Results from ISOQuant were further analyzed for changes in protein levels in $\mathrm{R} 3.6 .3$ with empirical Bayes method using package limma (XLSX)

Average expression, $p$-value, adjusted $p$-value, $t, B$, significance, and log FC values for hippocampus and rest of cerebrum (XLSX)

\section{AUTHOR INFORMATION}

\section{Corresponding Author}

Greta Hultqvist - Protein Drug Design, Faculty of Pharmacy, Biomedical Centre 591, Uppsala University, 75124 Uppsala, Sweden; (1) orcid.org/0000-0002-4136-6792; Phone: +46 70 2253522; Email: greta.hultqvist@farmbio.uu.se

\section{Authors}

Fadi Rofo - Protein Drug Design, Faculty of Pharmacy, Biomedical Centre 591, Uppsala University, 75124 Uppsala, Sweden

Friederike A. Sandbaumhüter - Medical Mass Spectrometry, Department of Pharmaceutical Biosciences, Biomedical Centre 591, Uppsala University, 75124 Uppsala, Sweden

Aikaterini Chourlia - Protein Drug Design, Faculty of Pharmacy, Biomedical Centre 591, Uppsala University, 75124 Uppsala, Sweden

Nicole G. Metzendorf - Protein Drug Design, Faculty of Pharmacy, Biomedical Centre 591, Uppsala University, 75124 Uppsala, Sweden

Jamie I. Morrison - Protein Drug Design, Faculty of Pharmacy, Biomedical Centre 591, Uppsala University, 75124 Uppsala, Sweden

Stina Syvänen - Department of Public Health and Caring Sciences, Rudbeck Laboratory, Uppsala University, 75185 Uppsala, Sweden; 이이.org/0000-0002-8196-4041

Per E. Andrén - Medical Mass Spectrometry, Department of Pharmaceutical Biosciences, Biomedical Centre 591 and Science for Life Laboratory, Spatial Mass Spectrometry, Biomedical Centre 591, Uppsala University, 75124 Uppsala, Sweden; () orcid.org/0000-0002-4062-7743

Erik T. Jansson - Medical Mass Spectrometry, Department of Pharmaceutical Biosciences, Biomedical Centre 591, Uppsala University, 75124 Uppsala, Sweden

Complete contact information is available at: https://pubs.acs.org/10.1021/acschemneuro.1c00303

\section{Author Contributions}

G.H., E.T.J., and P.E.A. designed the project. G.H. designed the protein. F.R. generated the recombinant protein. F.R., N.G.M., and S.S. carried out in vivo work. F.R. and F.A.S. prepared the tissues for LC-MS. F.A.S. performed the LCMS experiment. F.A.S. and E.T.J. analyzed LC-MS results. F.R., N.G.M., J.I.M., and A.C. performed the Western blot, MSD-multiplex, and ELISA assays. F.R., F.A.S., E.T.J., and G.H. wrote the manuscript with valuable inputs from the other coauthors.

\section{Notes}

The authors declare no competing financial interest.

The mass spectrometry proteomics data have been deposited to the ProteomeXchange Consortium via the PRIDE partner repository with the data set identifiers PXD022221 and
PXD026566. “dx.doi.org/10.6019/PXD022221 and dx.doi.org/10.6019/PXD026566”.

\section{ACKNOWLEDGMENTS}

The work in this paper was supported by research grants from the Swedish Research Council (Medicine and Health, 201803320, P.E.A.; 2019-01885, G.H.; 2017-02413, S.S.; Natural and Engineering Science, 2018-05501, P.E.A.; 2018-03988, E.T.J.); the Swedish Foundation for Strategic Research (RIF14-0078, P.E.A.; ICA16-0010, E.T.J.); the Swedish Brain Foundation (FO2018-0292, P.E.A.; ALZ2019-0029, S.S.); the Science for Life Laboratory (P.E.A.); the Jeanssons stiftelser, Åke Wibergs stiftelse (G.H. and E.T.J.); and the Alzheimerfonden (G.H.; S.S.), Hedlunds stiftelse (G.H.), Olle Engqvist stiftelse (G.H.), Åhlén-stiftelsen (G.H.; S.S.), Torsten Söderbergs stiftelse (G.H.), Magnus Bergvalls stiftelse (G.H.), Vinnova (G.H., S.S.). Schematic illustrations are created with Biorender.com. The animal work in this study was performed at the SciLifeLab Pilot Facility for Preclinical PET-MRI, a Swedish nationally available imaging platform at Uppsala University, Sweden, financed by the Knut and Alice Wallenberg Foundation.

\section{ABBREVIATIONS}

$\mathrm{A} \beta$, amyloid- $\beta$; APLP-1, amyloid-like protein 1 ; APP, amyloid precursor protein; $\mathrm{BBB}$, blood-brain barrier; FDR, false discovery rate; LC-MS, liquid chromatography-mass spectrometry; scFv, single chain fragment variable; SNARE, soluble $\mathrm{N}$-ethylmaleimide-sensitive fusion protein attachment protein receptor; SST, somatostatin; TfR, transferrin receptor; WT, wild-type

\section{REFERENCES}

(1) Murphy, M. P., and LeVine, H. (2010) Alzheimer's Disease and the Amyloid- $\beta$ Peptide. J. Alzheimer's Dis. 19 (1), 311-23.

(2) Haass, C., Kaether, C., Thinakaran, G., and Sisodia, S. (2012) Trafficking and proteolytic processing of APP. Cold Spring Harbor Perspect. Med. 2 (5), a006270.

(3) Iwata, N., Tsubuki, S., Takaki, Y., Watanabe, K., Sekiguchi, M., and Hosoki, E. (2000) Identification of the major Abeta1-42degrading catabolic pathway in brain parenchyma: suppression leads to biochemical and pathological deposition. Nat. Med. 6 (2), 143-50.

(4) Iwata, N., Tsubuki, S., Takaki, Y., Shirotani, K., Lu, B., and Gerard, N. P. (2001) Metabolic Regulation of Brain A $\beta$ by Neprilysin. Science 292 (5521), 1550-2.

(5) Carpentier, M., Robitaille, Y., DesGroseillers, L., Boileau, G., and Marcinkiewicz, M. (2002) Declining Expression of Neprilysin in Alzheimer Disease Vasculature: Possible Involvement in Cerebral Amyloid Angiopathy. J. Neuropathol. Exp. Neurol. 61 (10), 849-56.

(6) Rofo, F., Ugur Yilmaz, C., Metzendorf, N., Gustavsson, T., Beretta, C., Erlandsson, A., et al. (2021) Enhanced neprilysinmediated degradation of hippocampal A $\beta 42$ with a somatostatin peptide that enters the brain. Theranostics 11 (2), 789-804.

(7) O’Toole, T. J., and Sharma, S. (2020) Physiology, Somatostatin. StatPearls, 1.

(8) Saito, T., Iwata, N., Tsubuki, S., Takaki, Y., Takano, J., and Huang, S.-M. (2005) Somatostatin regulates brain amyloid beta peptide Abeta42 through modulation of proteolytic degradation. Nat. Med. 11 (4), 434-9.

(9) Martel, G., Dutar, P., Epelbaum, J., and Viollet, C. P. (2012) Somatostatinergic systems: an update on brain functions in normal and pathological aging. Front. Endocrinol. 3, 3.

(10) Ramos, B., Baglietto-Vargas, D., del Rio, J. C., MorenoGonzalez, I., Santa-Maria, C., and Jimenez, S. (2006) Early neuropathology of somatostatin/NPY GABAergic cells in the 
hippocampus of a PS1xAPP transgenic model of Alzheimer's disease. Neurobiol. Aging 27 (11), 1658-72.

(11) Reilly, J. F., Games, D., Rydel, R. E., Freedman, S., Schenk, D., and Young, W. G. (2003) Amyloid deposition in the hippocampus and entorhinal cortex: quantitative analysis of a transgenic mouse model. Proc. Natl. Acad. Sci. U. S. A. 100 (8), 4837-42.

(12) Fang, X. T., Hultqvist, G., Meier, S. R., Antoni, G., Sehlin, D., and Syvänen, S. (2019) High detection sensitivity with antibodybased PET radioligand for amyloid beta in brain. NeuroImage 01 (184), 881-8.

(13) Hultqvist, G., Syvänen, S., Fang, X. T., Lannfelt, L., and Sehlin, D. (2017) Bivalent Brain Shuttle Increases Antibody Uptake by Monovalent Binding to the Transferrin Receptor. Theranostics 7 (2), 308-18.

(14) Syvänen, S., Hultqvist, G., Gustavsson, T., Gumucio, A., Laudon, H., and Söderberg, L. (2018) Efficient clearance of $\mathrm{A} \beta$ protofibrils in $\mathrm{A} \beta \mathrm{PP}$-transgenic mice treated with a brain-penetrating bifunctional antibody. Alzheimer's Res. Ther. 10 (1), 49.

(15) Heneka, M. T., Carson, M. J., El Khoury, J., Landreth, G. E., Brosseron, F., Feinstein, D. L., et al. (2015) Neuroinflammation in Alzheimer's disease. Lancet Neurol. 14 (4), 388-405.

(16) Yang, Y., Kim, J., Kim, H. Y., Ryoo, N., Lee, S., and Kim, Y. (2015) Amyloid- $\beta$ oligomers may impair SNARE-mediated exocytosis by direct binding to syntaxin 1a. Cell Rep. 12 (8), 1244-51.

(17) Sokol, C. L., and Luster, A. D. (2015) The Chemokine System in Innate Immunity. Cold Spring Harbor Perspect. Biol. 7 (5), 7.

(18) Bayes-Genis, A., Barallat, J., and Richards, A. M. (2016) A Test in Context: Neprilysin: Function, Inhibition, and Biomarker. J. Am. Coll. Cardiol. 68 (6), 639-53.

(19) Jefferies, W. A., Brandon, M. R., Hunt, S. V., Williams, A. F., Gatter, K. C., and Mason, D. Y. (1984) Transferrin receptor on endothelium of brain capillaries. Nature 312 (5990), 162-3.

(20) Hauptmann, S., Scherping, I., Dröse, S., Brandt, U., Schulz, K. L., and Jendrach, M. (2009) Mitochondrial dysfunction: an early event in Alzheimer pathology accumulates with age in $\mathrm{AD}$ transgenic mice. Neurobiol. Aging 30 (10), 1574-86.

(21) Caspersen, C., Wang, N., Yao, J., Sosunov, A., Chen, X., Lustbader, J. W., et al. (2005) Mitochondrial A $\beta$ : a potential focal point for neuronal metabolic dysfunction in Alzheimer's disease. FASEB J. 19 (14), 2040-1.

(22) Lustbader, J. W., Cirilli, M., Lin, C., Xu, H. W., Takuma, K., and Wang, N. (2004) ABAD directly links Abeta to mitochondrial toxicity in Alzheimer's disease. Science 304 (5669), 448-52.

(23) Chen, L., Xu, S., Wu, T., Shao, Y., Luo, L., and Zhou, L. (2019) Studies on APP metabolism related to age-associated mitochondrial dysfunction in APP/PS1 transgenic mice. Aging 11 (22), 10242-51. (24) Reddy, P. H., and Beal, M. F. (2008) Amyloid beta, mitochondrial dysfunction and synaptic damage: implications for cognitive decline in aging and Alzheimer's disease. Trends Mol. Med. 14 (2), 45-53.

(25) Lovell, M. A., Xie, C., Gabbita, S. P., and Markesbery, W. R. (2000) Decreased thioredoxin and increased thioredoxin reductase levels in alzheimer's disease brain. Free Radical Biol. Med. 28 (3), 418-27.

(26) Rhein, V., Song, X., Wiesner, A., Ittner, L. M., Baysang, G., and Meier, F. (2009) Amyloid- $\beta$ and tau synergistically impair the oxidative phosphorylation system in triple transgenic Alzheimer's disease mice. Proc. Natl. Acad. Sci. U. S. A. 106 (47), 20057-62.

(27) Solarski, M., Williams, D., Mehrabian, M., Wang, H., Wille, H., and Schmitt-Ulms, G. (2019) The human brain somatostatin interactome: SST binds selectively to P-type family ATPases. PLoS One 14 (5), No. e0217392.

(28) Grois, L., Hupf, J., Reinders, J., Schröder, J., Dietl, A., and Schmid, P. M. (2017) Combined Inhibition of the Renin-Angiotensin System and Neprilysin Positively Influences Complex Mitochondrial Adaptations in Progressive Experimental Heart Failure. PLoS One 12 (1), 1 .
(29) Johnson, R. J., Xiao, G., Shanmugaratnam, J., and Fine, R. E. (2001) Calreticulin functions as a molecular chaperone for the $\beta$ amyloid precursor protein. Neurobiol. Aging 22 (3), 387-95.

(30) Pardossi-Piquard, R., Petit, A., Kawarai, T., Sunyach, C., Alves da Costa, C., and Vincent, B. (2005) Presenilin-Dependent Transcriptional Control of the A $\beta$-Degrading Enzyme Neprilysin by Intracellular Domains of $\beta$ APP and APLP. Neuron 46 (4), 541-54.

(31) Bayer, T. A., Paliga, K., Weggen, S., Wiestler, O. D., Beyreuther, K., and Multhaup, G. (1997) Amyloid precursor-like protein 1 accumulates in neuritic plaques in Alzheimer's disease. Acta Neuropathol. 94 (6), 519-24.

(32) Yanagida, K., Okochi, M., Tagami, S., Nakayama, T., Kodama, T. S., and Nishitomi, K. (2009) The 28-amino acid form of an APLP1-derived $\mathrm{A} \beta$-like peptide is a surrogate marker for $\mathrm{A} \beta 42$ production in the central nervous system. EMBO Mol. Med. 1 (4), 223-35.

(33) Sjödin, S., Andersson, K. K. A., Mercken, M., Zetterberg, H., Borghys, H., and Blennow, K. (2015) APLP1 as a cerebrospinal fluid biomarker for $\gamma$-secretase modulator treatment. Alzheimer's Res. Ther. 7 (1), 77.

(34) Nag, S., Yee, B. K., and Tang, F. (1999) Reduction in somatostatin and substance $\mathrm{P}$ levels and choline acetyltransferase activity in the cortex and hippocampus of the rat after chronic intracerebroventricular infusion of beta-amyloid (1-40). Brain Res. Bull. 50 (4), 251-62.

(35) Hickman, S. E., Kingery, N. D., Ohsumi, T. K., Borowsky, M. L., Wang, L., and Means, T. K. (2013) The microglial sensome revealed by direct RNA sequencing. Nat. Neurosci. 16 (12), 1896905.

(36) Serdar, M., Kempe, K., Herrmann, R., Picard, D., Remke, M., Herz, J., et al. (2020) Involvement of CXCL1/CXCR2 During Microglia Activation Following Inflammation-Sensitized HypoxicIschemic Brain Injury in Neonatal Rats. Front Neurol 11, 11.

(37) Zhang, K., Tian, L., Liu, L., Feng, Y., Dong, Y.-B., Li, B., et al. (2013) CXCL1 contributes to $\beta$-amyloid-induced transendothelial migration of monocytes in Alzheimer's disease. PLoS One 8 (8), e72744.

(38) Johnson, E. A., Dao, T. L., Guignet, M. A., Geddes, C. E., Koemeter-Cox, A. I., and Kan, R. K. (2011) Increased expression of the chemokines CXCL1 and MIP- $1 \alpha$ by resident brain cells precedes neutrophil infiltration in the brain following prolonged somaninduced status epilepticus in rats. J. Neuroinflammation 8 (1), 41.

(39) Ramakrishnan, N. A., Drescher, M. J., and Drescher, D. G. (2012) The SNARE complex in neuronal and sensory cells. Mol. Cell. Neurosci. 50 (1), 58-69.

(40) Gautam, V., D’Avanzo, C., Berezovska, O., Tanzi, R. E., and Kovacs, D. M. (2015) Synaptotagmins interact with APP and promote $\mathrm{A} \beta$ generation. Mol. Neurodegener. 10, 31 .

(41) Wong, S. S., Sun, N. N., Fastje, C. D., Witten, M. L., Lantz, R. C., and Lu, B. (2011) Role of Neprilysin in Airway Inflammation Induced by Diesel Exhaust Emissions. Res. Rep. Health Eff Inst. 159, $3-40$.

(42) Suh, J., Romano, D. M., Nitschke, L., Herrick, S. P., DiMarzio, B. A., Dzhala, V., et al. (2019) Loss of Ataxin-1 Potentiates Alzheimer's Pathogenesis by Elevating Cerebral BACE1 Transcription. Cell 178 (5), 1159-1175.

(43) Liu, R., and Tian, Q. (2009) Protein phosphatase 2A, a key player in Alzheimer's disease. Front Med. China. 3 (1), 8-12.

(44) Sontag, J-M, and Sontag, E. (2014) Protein phosphatase 2A dysfunction in Alzheimer's disease. Front. Mol. Neurosci. 7, 7.

(45) Kakiya, N., Saito, T., Nilsson, P., Matsuba, Y., Tsubuki, S., Takei, N., et al. (2012) Cell-surface expression of the major $\mathrm{A} \beta$ degrading enzyme, neprilysin, depends on phosphorylation by MEK and dephosphorylation by protein phosphatase 1a. J. Biol. Chem. 5, 29362.

(46) Wildasin, K. (2004) Role of reticulon proteins in Alzheimer's disease. Lancet Neurol. 3 (10), 576.

(47) Manczak, M., Kandimalla, R., Fry, D., Sesaki, H., and Reddy, P. H. (2016) Protective effects of reduced dynamin-related protein 1 
against amyloid beta-induced mitochondrial dysfunction and synaptic damage in Alzheimer's disease [Internet]. Hum. Mol. Genet. 25, 1.

(48) Zhu, L., Su, M., Lucast, L., Liu, L., Netzer, W. J., and Gandy, S. E. (2012) Dynamin 1 Regulates Amyloid Generation through Modulation of BACE-1. PLoS One 7 (9), e45033.

(49) Nilsson, P., Loganathan, K., Sekiguchi, M., Winblad, B., Iwata, N., Saido, T. C., et al. (2015) Loss of neprilysin alters protein expression in the brain of Alzheimer's disease model mice. Proteomics 15 (19), 3349-55.

(50) Fan, F., Funk, L., and Lou, X. (2016) Dynamin 1- and 3Mediated Endocytosis Is Essential for the Development of a Large Central Synapse In Vivo. J. Neurosci. 36 (22), 6097.

(51) Dickinson, R. J., and Keyse, S. M. (2006) Diverse physiological functions for dual-specificity MAP kinase phosphatases. J. Cell Sci. 119 (22), 4607-15.

(52) Taylor, D. M., Moser, R., Régulier, E., Breuillaud, L., Dixon, M., and Beesen, A. A. (2013) MAP kinase phosphatase 1 (MKP-1/ DUSP1) is neuroprotective in Huntington's disease via additive effects of JNK and p38 inhibition. J. Neurosci. 33 (6), 2313-25.

(53) Sontag, E., Nunbhakdi-Craig, V., Sontag, J.-M., Diaz-Arrastia, R., Ogris, E., and Dayal, S. (2007) Protein phosphatase 2A methyltransferase links homocysteine metabolism with tau and amyloid precursor protein regulation. J. Neurosci. 27 (11), 2751-9.

(54) Distler, U., Kuharev, J., Navarro, P., and Tenzer, S. (2016) Label-free quantification in ion mobility-enhanced data-independent acquisition proteomics. Nat. Protoc. 11 (4), 795-812.

(55) Distler, U., Kuharev, J., Navarro, P., Levin, Y., Schild, H., and Tenzer, S. (2014) Drift time-specific collision energies enable deepcoverage data-independent acquisition proteomics. Nat. Methods 11 (2), 167-70.

(56) Ritchie, M. E., Phipson, B., Wu, D., Hu, Y., Law, C. W., and Shi, W. (2015) limma powers differential expression analyses for RNAsequencing and microarray studies. Nucleic Acids Res. 43 (7), e47.

(57) Storey, J. D., and Tibshirani, R. (2003) Statistical significance for genomewide studies. Proc. Natl. Acad. Sci. U. S. A. 100 (16), $9440-5$. 